\title{
Enhanced antioxidant bioactivity of Salvia miltiorrhiza (Danshen) products prepared using nanotechnology
}

\author{
Je-Ruei Liu ${ }^{\mathrm{a}}$, Guo-Feng Chen ${ }^{\mathrm{b}}$, Hui-Nung Shih ${ }^{\mathrm{b}}$, Ping-Chung Kuo ${ }^{\mathrm{b}, *}$ \\ a Department of Animal Science and Technology and Institute of Biotechnology, National Taiwan University, \\ Taipei 102, Taiwan, ROC \\ ${ }^{\mathrm{b}}$ Department of Biotechnology, National Formosa University, 64 Wunhua Road, Huwei Town, Yunlin 632, Taiwan, ROC
}

\begin{abstract}
The traditional Chinese medicine, Salvia miltiorrhiza (Danshen), promotes blood circulation and relieves blood stasis, also demonstrating good antioxidant activity. In the present study, therefore, the antioxidant activities of medicinal plant materials prepared using nanotechnology or traditional grinding methods were compared using three biological assays. It was found that the nanotechnology preparation had stronger antioxidant bioactivities. Complementary quantitative analysis of four active constituents, salvianolic acid B, cryptotanshinone, tanshinone I and tanshinone IIA, by HPLC revealed only marked differences for salvianolic acid B. The results indicate that the polar active constituent in the nanotechnology samples was released faster compared to the traditionally powdered samples.
\end{abstract}

(C) 2007 Elsevier GmbH. All rights reserved.

Keywords: Traditional Chinese medicine; Antioxidant activity; HPLC; Salvianolic acid B

\section{Introduction}

Nanoscience may be defined as the extension of existing sciences into dimensional realms much smaller than previously considered feasible, with nanotechnology representing the practical application thereof. Current nanotechnology developments have led to nanomedicine, a new field which includes many diagnostic and therapeutic applications involving nanomaterials and nanodevices (Kagan et al., 2005). It has been envisioned that nanomedical products will have a huge impact on public health care (Zajtchuk, 1999). Among the many nanoscience breakthroughs in recent years, potential applications include toxicity reduction where the focus has been on decreasing drug concentration in

\footnotetext{
${ }^{*}$ Corresponding author. Tel.: + 88656315491 ; fax: +88656315502.

E-mail address: pcckuoo@sunws.nfu.edu.tw (P.-C. Kuo).
}

overdose cases, for example, the tricyclic antidepressants (Ma and Henry, 2001; Gabel and Hinkelbein, 2004; Brucculeri et al., 2005). Superior performance has been demonstrated for some approaches to detoxification therapy using nanoparticles and nanoemulsions (Morey et al., 2004; Varshney et al., 2004) relative to their macro-scale counterparts. However, interest in the application of nanotechnology to preparation of traditional Chinese medicine is comparatively low, mainly for two reasons. Firstly, the modest cost of traditional Chinese medicine reduces the economic benefit of utilizing nanotechnology. Secondly, the toxicity of the traditional Chinese medicines used in the common medicinal preparations is relatively low. However, as global climate problems increasingly draw attention to environmental issues, interest in natural solutions has also grown. Since most of the important plant materials used in traditional Chinese medicine are cultivated in 
regions such as the Inner Mongolian grassland that attract environmental concerns, the large-scale harvest of the raw plant materials causes more and more serious environmental problems, e.g., dust storms. Hence, application of nanotechnology to commonly used traditional Chinese medicine plant materials may not only improve their bioactivity but also reduce the amount of the nanopharmaceuticals required and, thereby, decrease environmental degradation associated with the harvesting of the raw products.

Many reactive oxygen species (ROS), including the hydroxyl radical, hydrogen peroxide and the peroxide radical, are known to cause oxidative damage to living systems. ROS also play a significant role in human diseases such as cancer, atherosclerosis, hypertension and arthritis (Halliwell and Gutteridge, 1984; Frenkel, 1992). Given this negative impact, there is increasing interest in discovering natural antioxidants derived from traditional Chinese medicines as it is believed that they protect the human body from the attack of free radicals and retard the progress of many chronic diseases (Pryor, 1991). Further, these natural antioxidants are likely to be more desirable than chemically produced analogs because some of the latter are reportedly carcinogenic (Imaida et al., 1983).

The dried roots of Salvia miltiorrhiza (Danshen) are widely used as traditional Chinese medicines for promoting circulation and improving blood stasis. Danshen is also extensively used for the treatment and prevention of coronary heart and cerebrovascular diseases, and hyperlipidemia (Zhou et al., 2006; Kang et al., 2004). In a survey of the relevant literature, strong protective action, against oxidative damage to liver microsomes, hepatocytes and erythrocytes, had been demonstrated for seven phenolic compounds isolated from $S$. miltiorrhiza as active components (Liu et al., 1992). Of these, significant antioxidant activities have been demonstrated for its major component, salvianolic acid B (Kang et al., 2004). In the present study, therefore, Danshen powders prepared using nanotechnology and traditional grinding methods were evaluated to compare their antioxidant power and salvianolic acid $\mathrm{B}$ content and, thus, determine the relative bioactivities of the two plant material variants.

\section{Materials and methods}

\section{General experimental procedures}

All chemicals were purchased from Sigma-Aldrich Co., USA, unless otherwise indicated. The HPLC-grade methanol and acetonitrile were purchased from E. Merck Co., USA. Plant materials were extracted using a Major Science LM-570R shaking incubator. UV and visible spectra were acquired on a GBC Cintra 101 $\mathrm{UV}-\mathrm{V}$ is spectrophotometer. The optical density (OD) at various wavelengths was determined using a $\mu$ Quant universal microplate spectrophotometer. The transmission electron microscopy (TEM) was undertaken with a JEOL JEM-1400 Transmission Electron Microscope. High-performance liquid chromatography (HPLC) was performed on a Shimadzu LC-10AT $\mathrm{VP}$ series pumping system equipped with a Shimadzu SPD-M10A $A_{V P}$ UVVis detector, a Merck Lichrospher ${ }^{\circledR} 100$ RP-18 $(5 \mu \mathrm{m})$ column, and a Rheodyne injector.

\section{Plant material}

The dried roots of $S$. miltiorrhiza were purchased form herbal markets in Nantou, Taiwan. The voucher specimen was deposited in the herbarium of the Diamond Nano-Biochem Company, Taichung, Taiwan. All the Danshen powder, prepared by either traditional grinding or nanotechnology, was generously provided by Diamond Nano-Biochem Company. The dried roots of $S$. miltiorrhiza were ground by the atomizer and further sprayed granulating with the aid of floating bed. The resulting materials were dried to form the nanoparticles under the dry processes (Wang, 2007). The Danshen powder prepared by nanotechnology was analyzed by transmission electron microscopy (TEM) to certify particle size in the nanoscale range.

\section{Transmission electron microscopy (TEM)}

Contrast for TEM was achieved by adding 1-dodecane $(20 \%, w)$ in major oil (ethyl butyrate) as a dopant to the nanocapsule oil core. A 1000-fold dilution of stock nanocapsule solution was dripped onto a carbon-coated gold grid, and left overnight to allow moisture evaporation. The grids were then placed in a sealed container where $\mathrm{OsO}_{4}$ vapor was diffused through the polysiloxane/silicate to stain the 1-dodecane in the nanocapsule core for not less than $4 \mathrm{~h}$. TEM images were obtained at $75 \mathrm{kV}$. Data were compared with images of three independently made formulations.

\section{Sample preparations for Danshen powder}

Danshen powder $(1.0 \mathrm{~g})$ prepared by traditional grinding or nanotechnology (Diamond Nano-Biochem Co.) was extracted with $10 \mathrm{ml}$ methanol in a $100 \mathrm{ml}$ flask. The samples were extracted in a shaking incubator at $25^{\circ} \mathrm{C}$ and $150 \mathrm{rpm}$ for $10,30,50,70$ or $90 \mathrm{~min}$. The final volume was raised to $50 \mathrm{ml}$ after the solution was filtered and kept at $4{ }^{\circ} \mathrm{C}$ for the analyses. The sample solution was then either filtered through a membrane $(0.45 \mu \mathrm{m})$ before injection into a HPLC to quantifying the cryptotanshinone, tanshinone IIA, tanshinone I and 
salvianolic acid $\mathrm{B}$, or assigned to determine the antioxidant activities, including 1,1-diphenyl- $\beta$-picrylhydrazyl (DPPH) radical scavenging activity, ferrous ion chelating ability and reducing power.

\section{Chromatography}

The elution gradient for the HPLC analysis consisted of two solvent compositions: $0.5 \%$ acetic acid in acetonitrile (solvent $\mathrm{A}$ ) and $0.5 \%$ acetic acid in water (solvent B). Gradient elution for solvent A was as follows: maintenance at a concentration of $15 \%$ for the first $15 \mathrm{~min}$, then increase to $47 \%$ in the next $20 \mathrm{~min}$, hold for $15 \mathrm{~min}$ before further increase to $65 \%$ in the next $15 \mathrm{~min}$, then decrease to $50 \%$ in $15 \mathrm{~min}$, return to $15 \%$ in the next $5 \mathrm{~min}$ and hold for $45 \mathrm{~min}$ for the next injection $(100 \mu \mathrm{l}$ each). The column and auto-sampler were set at ambient temperature; flow rate was $0.5 \mathrm{ml} / \mathrm{min}$. The eluent was monitored by a UV detector at $280 \mathrm{~nm}$ for all the studied compounds.

\section{Preparation of standard solutions and calibration curves}

The reference compounds, cryptotanshinone, tanshinone IIA, tanshinone I and salvianolic acid B were purchased from China Chengdu Scholar Bio-tech Company. The methanolic standard solutions were prepared as follows: $100.0 \mu \mathrm{g} / \mathrm{ml}$ of cryptotanshinone and tanshinone IIA; $20.0 \mu \mathrm{g} / \mathrm{ml}$ of tanshinone I; and, $1.0 \mathrm{mg} / \mathrm{ml}$ of salvianolic acid B. Standard stock solution containing cryptotanshinone, tanshinone IIA, tanshinone I and salvianolic acid B was prepared by mixing and diluting the original stock solution with $0.5 \%$ acetic acid in methanol/water (1:1) to achieve final concentrations of $12.0,6.0,6.0$ and $100.0 \mu \mathrm{g} / \mathrm{ml}$, respectively. Working standard solutions and quality control samples at high, medium and low concentrations were prepared by diluting the stock solution with $0.5 \%$ acetic acid in methanol/water (1:1). These solutions were all kept in brown glass bottles and stored at $-20^{\circ} \mathrm{C}$.

Calibration curves were constructed by plotting the peak area versus analyte standard solutions at various concentrations. The studied concentration ranges were $0.3-9.0 \mu \mathrm{g} / \mathrm{ml}$ for cryptotanshinone, $0.15-4.5 \mu \mathrm{g} / \mathrm{ml}$ for both tanshinone IIA and tanshinone I, and $33.8-338 \mu \mathrm{g} / \mathrm{ml}$ for salvianolic acid B.

\section{Radical scavenging effect upon 1,1-diphenyl-2- picrylhydrazyl (DPPH) radicals}

The DPPH assay was based on the reported method (Shimada et al., 1992). A methanol solution of $200 \mu \mathrm{M}$ of the chemically stable free radical 1,1-diphenyl-2picrylhydrazyl was incubated with an equal volume of antioxidant for $30 \mathrm{~min}$. The DPPH radical scavenging activities of Danshen samples for different extraction periods were measured after 40-fold dilution of the prepared sample solutions and expressed in terms of inhibition percentage $=[1-($ absorbance of samples at $517 \mathrm{~nm}) /($ absorbance of control at $517 \mathrm{~nm})] \times 100(\%)$.

\section{Chelation of ferrous ions}

The chelation of ferrous ions by the sample was estimated using the reported method (Yen et al., 2000). Briefly, tested sample solutions $(0.2 \mathrm{ml}$, five-fold dilution of prepared sample) were added to a solution of $2.0 \mathrm{mM}$ ferrous chloride $(0.1 \mathrm{ml})$ and $3.7 \mathrm{ml}$ methanol. The reaction was initiated by addition of $5 \mathrm{mM}$ ferrozine $(0.2 \mathrm{ml})$, with the mixture then shaken vigorously and left standing at room temperature for $10 \mathrm{~min}$. The absorbance of the resulting solution was measured at $562 \mathrm{~nm}$. The relative capability of the sample to chelate the ferrous ions was derived from the formula: [1-(sample absorbance at $562 \mathrm{~nm}) /($ control absorbance at $562 \mathrm{~nm})] \times 100(\%)$. Higher percentage indicates greater chelating ability.

\section{Reducing power}

The reducing power of each extract was determined according to the reported method (Liu et al., 2005). Sample solutions $(2.5 \mathrm{ml}$, five-fold dilution of prepared sample) in phosphate buffer $(2.5 \mathrm{ml}, 0.2 \mathrm{M}, \mathrm{pH} 6.6)$ were added to potassium ferricyanide $(2.5 \mathrm{ml}, 1.0 \%)$, and the mixture was incubated at $50{ }^{\circ} \mathrm{C}$ for $20 \mathrm{~min}$. Trichloroacetic acid $(2.5 \mathrm{ml}, 10 \%)$ was added and the mixture was centrifuged at $650 \mathrm{~g}$ for $10 \mathrm{~min}$. The supernatant $(5.0 \mathrm{ml})$ was mixed with distilled water $(5.0 \mathrm{ml})$ and ferric chloride $(1.0 \mathrm{ml}, 0.1 \%)$, and then the solution's absorbance of the incident radiation was determined spectrophotometrically at $700 \mathrm{~nm}$, with higher absorbance value indicating greater reducing power.

\section{Statistical analysis}

Statistical Analysis System software (version 6.1; SAS, 1998) was used to test the significance of all results. Measurement results are expressed as mean \pm standard deviation (SD). The student's $t$ test was applied for inter-group comparison, with $p<0.05$ considered significant for all tests.

\section{Results}

The dried roots of $S$. miltiorrhiza were purchased and prepared by traditional methods or nanotechnology. The particle size of the traditionally ground samples was 
around $300 \mu \mathrm{m}$. Most of the nanocapsules were less than $100 \mathrm{~nm}$ in size, however, some aggregation still occurred (representative TEM shown in Fig. 1). Cellular disruption was evident in the tested study samples prepared by nanotechnology. Although the transmission electron microscopy was only used to test a small part of the overall sample, the results obtained were in good agreement with the generality (Jovanovic et al., 2006).

The DPPH radical scavenging percentage for the Danshen samples prepared by traditional grinding and nanotechnology for various extraction periods are plotted in Fig. 2. The analytical data show that the Danshen samples prepared using nanotechnology all exhibited significantly more scavenging power compared to the traditionally ground analogs. Additionally, the chelation of ferrous ions was examined using the

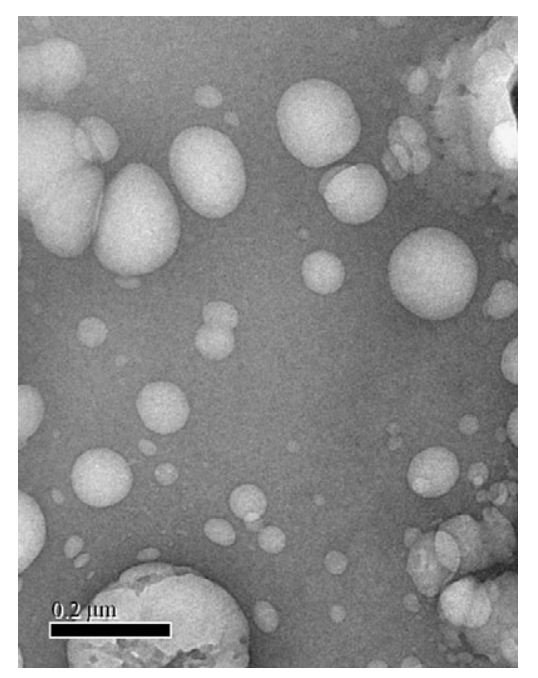

Fig. 1. Transmission electron microscopy image of Danshen samples prepared by nanotechnology. previously reported methods, with chelation percentages for different extraction periods shown in Fig. 3. The inhibition percentages for the nanoscale samples were markedly higher than the traditionally ground analogs, thus indicating stronger ferrous ion chelation for the former. The reducing power for the two preparation methods is compared in Fig. 4. The reducing power of the two Danshen preparations also exhibited same results as in the examinations of DPPH radical scavenging and ferrous ions chelating assays. The reducing power of nanosized samples was also significantly higher than the common preparations.

For elucidating the relationship between antioxidant activity and particle size, it is important to determine the differences in the composition of the studied preparation methods. Hence, high-performance liquid chromatography (HPLC) was applied to evaluate the contents of the major constituents in the two samples. Gradient elution with $0.5 \%$ acetic acid in acetonitrile and water was performed according to the reported method (Liu et al., 2006). Typical chromatogram for standard solution of the four major components, salvianolic acid B, cryptotanshinone, tanshinone I and tanshinone IIA, is presented in Fig. 5, with retention times of 34.3, 74.1, 75.2 and $87.8 \mathrm{~min}$, respectively, under the same experimental conditions. As shown in Table 1, the calibration curves of the tested compounds were linear over the studied concentration ranges (correlation coefficient $r^{2}>0.997$ for the four analytes). The component contents in the Danshen samples prepared by nanotechnology and traditionally grinding are presented in Table 2. Interestingly, only salvianolic acid B concentration varied markedly between preparations. Although the concentration of salvianolic acid B was as high as $1.215 \mathrm{mg} / \mathrm{g}$ in the nanotechnology preparation after 10 min of extraction, the concentration did not increase

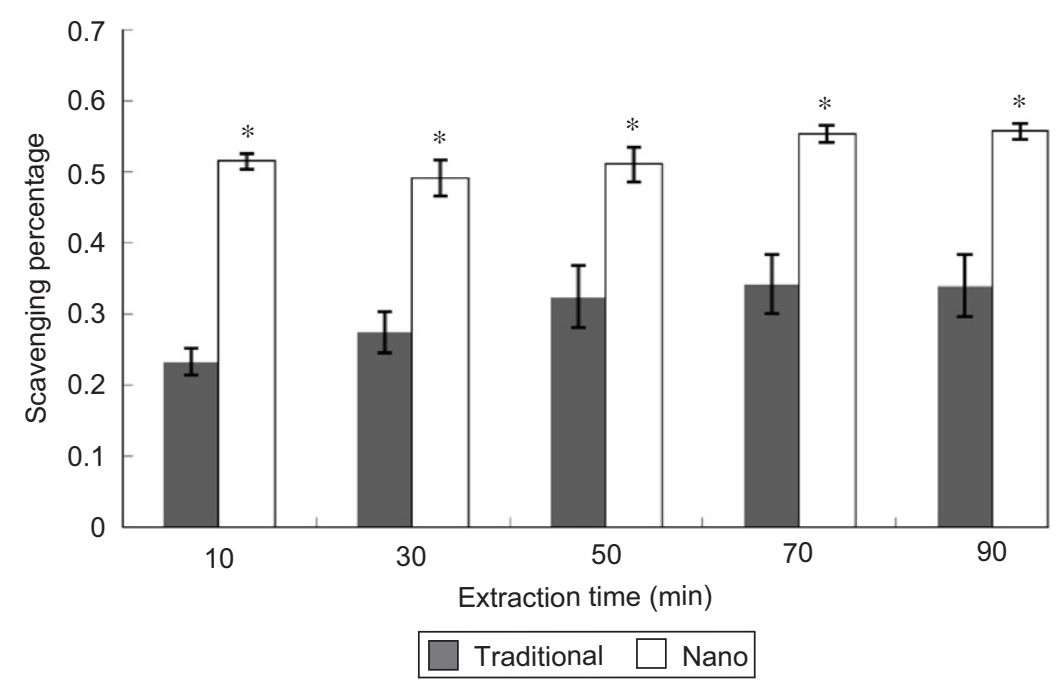

Fig. 2. DPPH radical scavenging activity of Danshen samples with different extraction times. *Significantly different from comparable values for traditionally grounded method. 


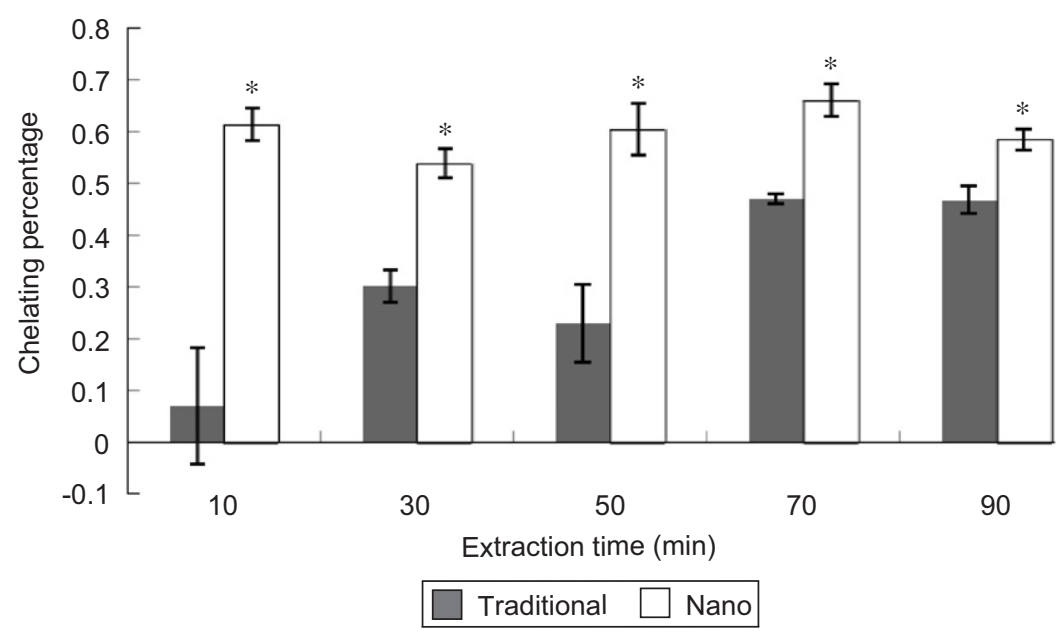

Fig. 3. Ferrous ions chelating ability of Danshen samples under different extraction times. *Significantly different from comparable values for traditionally grounded method.

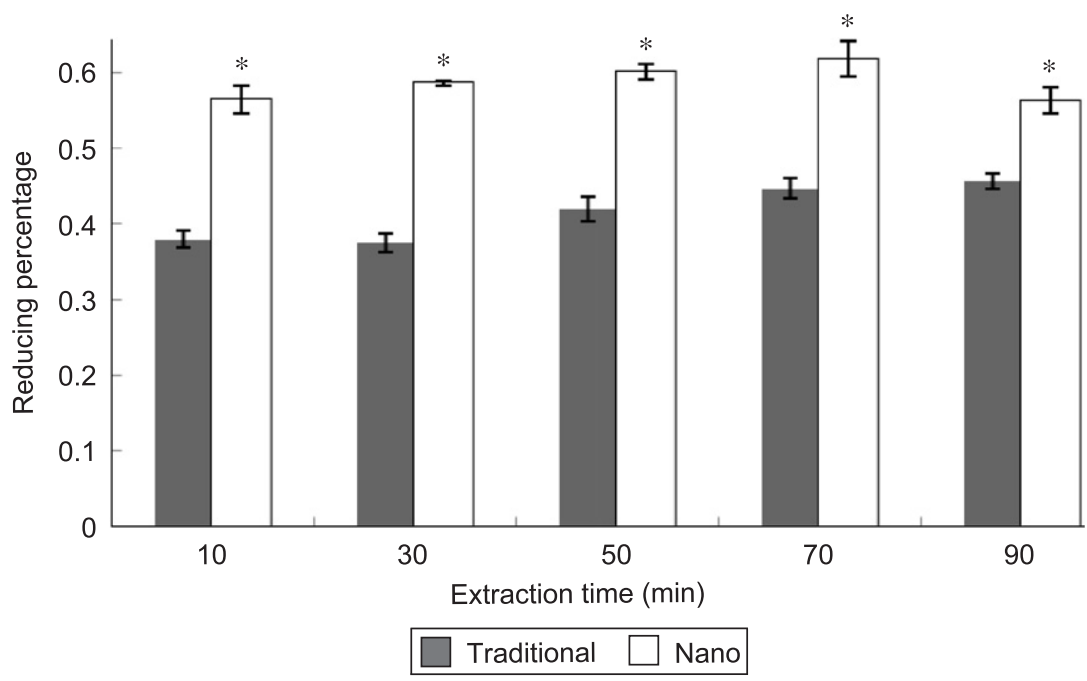

Fig. 4. Reducing power of Danshen samples under different extraction times. *Significantly different from comparable values for traditionally grounded method.

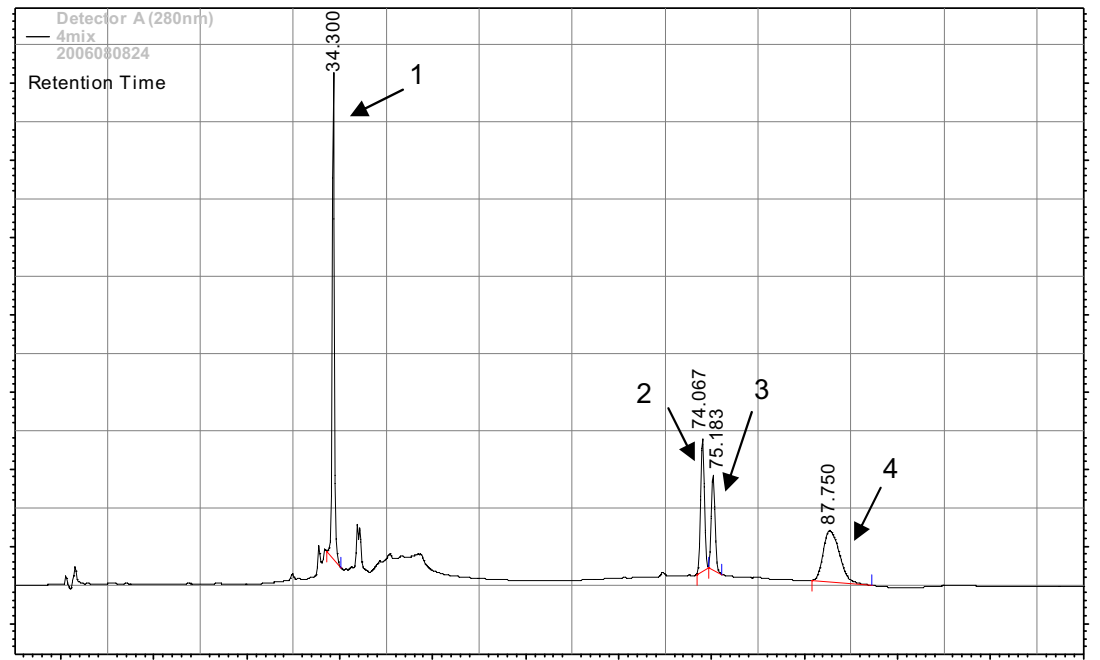

Fig. 5. HPLC chromatograms of the major components in standard solution. (1) salvianolic acid $B$, RT $=34.3$ min; (2) cryptotanshinone, RT = 74.1 $\mathrm{min}$; (3) tanshinone IIA, RT = 75.2 $\mathrm{min}$ and (4) tanshinone $\mathrm{I}$, RT $=87.8 \mathrm{~min}$. 
Table 1. Analytical data of the four tested compounds

\begin{tabular}{llll}
\hline Analyte & $\begin{array}{l}\text { Retention time } \\
(\mathrm{min})\end{array}$ & $\begin{array}{l}\text { Linear equation of calibration } \\
\text { curve }\end{array}$ & $\begin{array}{l}\text { Correlation } \\
\text { coefficient }\left(r^{2}\right)\end{array}$ \\
\hline Salvianolic acid B & 34.3 & $Y=7 \times 10^{6} X-79143$ & 0.9976 \\
Cryptotanshinone & 74.1 & $Y=19048 X+192$ & 0.9999 \\
Tanshinone I & 75.2 & $Y=44541 X-5488$ & 0.9996 \\
Tanshinone IIA & 87.8 & $Y=61560 X-1780$ & 0.9998 \\
\hline
\end{tabular}

Table 2. Concentrations of four components in the Danshen samples

\begin{tabular}{|c|c|c|c|c|c|}
\hline \multirow[t]{2}{*}{ Analyte } & \multicolumn{5}{|c|}{ Extraction time (min) } \\
\hline & 10 & 30 & 50 & 70 & 90 \\
\hline Salvianolic acid B & $\begin{array}{c}620.7 \pm 40.7 \\
1215.4 \pm 54.4^{*}\end{array}$ & $\begin{array}{c}781.1 \pm 52.8 \\
1269.4 \pm 46.9^{*}\end{array}$ & $\begin{array}{c}825.1 \pm 50.9 \\
1312.5 \pm 18.2^{*}\end{array}$ & $\begin{array}{c}861.2 \pm 46.1 \\
1277.8 \pm 57.7 *\end{array}$ & $\begin{array}{c}883.0 \pm 16.2 \\
1233.9 \pm 41.4^{*}\end{array}$ \\
\hline Cryptotanshinone & $\begin{array}{l}13.3 \pm 1.3 \\
16.7 \pm 2.0\end{array}$ & $\begin{array}{l}15.2 \pm 0.2 \\
15.7 \pm 1.6\end{array}$ & $\begin{array}{l}16.5 \pm 1.2 \\
14.8 \pm 3.1\end{array}$ & $\begin{array}{l}18.1 \pm 0.3 \\
15.0 \pm 1.0^{*}\end{array}$ & $\begin{array}{l}16.9 \pm 0.9 \\
14.6 \pm 1.3\end{array}$ \\
\hline Tanshinone I & $\begin{array}{l}17.5 \pm 1.0 \\
20.9 \pm 0.6^{*}\end{array}$ & $\begin{array}{l}19.7 \pm 1.6 \\
20.4 \pm 0.2\end{array}$ & $\begin{array}{l}21.1 \pm 2.2 \\
20.2 \pm 0.8\end{array}$ & $\begin{array}{l}21.3 \pm 1.0 \\
20.0 \pm 0.1\end{array}$ & $\begin{array}{l}21.8 \pm 1.0 \\
20.7 \pm 0.2\end{array}$ \\
\hline Tanshinone IIA & $\begin{array}{l}35.2 \pm 2.8 \\
37.6 \pm 0.7\end{array}$ & $\begin{array}{l}40.0 \pm 2.1 \\
36.7 \pm 1.5\end{array}$ & $\begin{array}{l}42.8 \pm 4.1 \\
37.0 \pm 1.7\end{array}$ & $\begin{array}{l}44.1 \pm 1.5 \\
36.8 \pm 1.7^{*}\end{array}$ & $\begin{array}{l}42.7 \pm 2.4 \\
37.2 \pm 2.9\end{array}$ \\
\hline
\end{tabular}

The concentrations were presented at $\mu \mathrm{g} / \mathrm{g}$ of danshen samples. The upper row data for each constituent meant the concentration in samples prepared by traditionally grounded method, and the other meant the concentration in samples prepared by nantotechnology. *Significantly different from comparable values for traditionally grounded method.

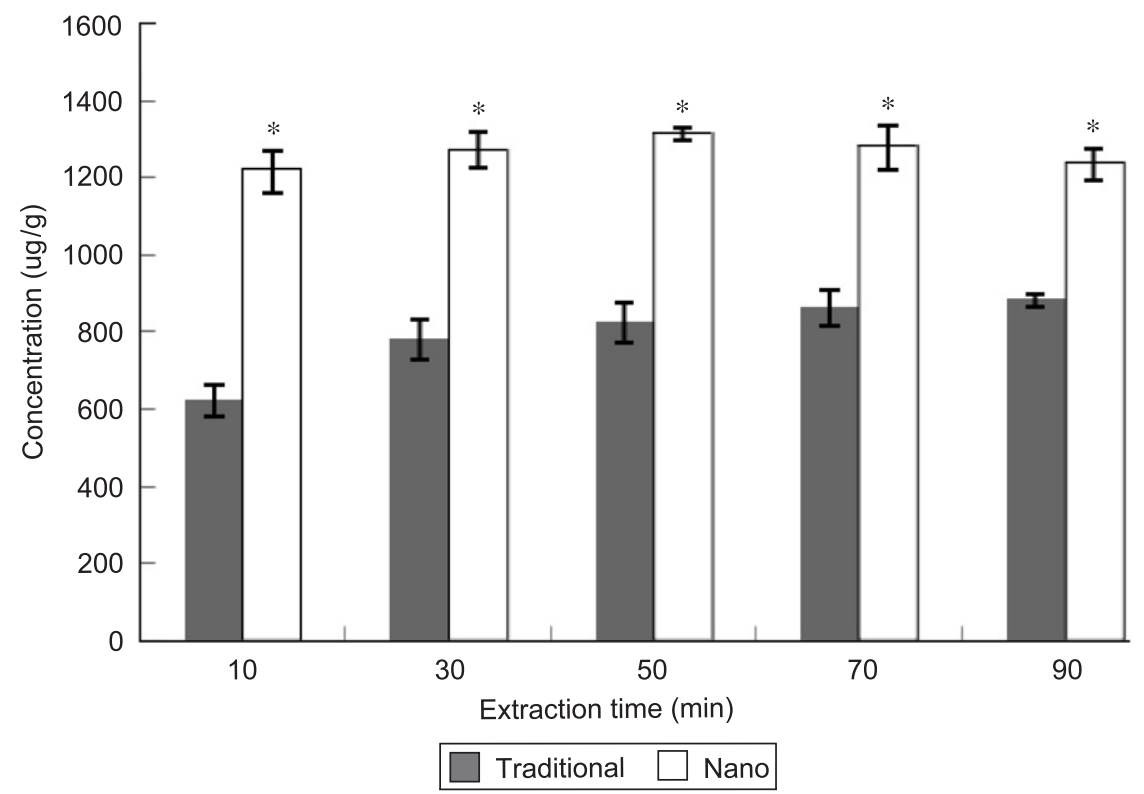

Fig. 6. Concentration differences of salvianolic acid between samples prepared by traditionally grounded method and by nanotechnology. *Significantly different from comparable values for traditionally grounded method.

with further extraction. By contrast, the concentration of salvianolic acid B increased with extraction time in samples powdered using the traditional procedure; however, the concentration did not exceed that of the nanotechnology samples even where the extraction time was extrapolated to infinity (Fig. 6). In addition, significant differences were not demonstrated comparing the levels of the other three components, cryptotanshinone, tanshinone I and 
tanshinone IIA, between the two studied preparation methods (Table 2).

\section{Discussion}

In the present study, the efficacies of medicinal plant materials prepared using nanotechnology or traditional grinding were compared by examining antioxidant activity through three biological assays measuring DPPH free radical scavenging, ferrous ion chelation, and reduction power. Stronger antioxidant bioactivities were observed for the extracts prepared using nanotechnology in all tested assays. These results suggest greater liberation of active components in the Danshen samples prepared using the modern technique. According to pharmacological investigation, the active constituents of Danshen can be divided into two groups: hydrophilic (e.g., phenolic acids) and lipophilic (e.g., tanshinones) (Kang et al., 2004; Zhou et al., 2006; Liu et al., 2006). Various in vitro and in vivo pharmacological experiments have demonstrated that both hydrophilic and lipophilic compounds can improve microcirculation, increase the blood flow and prevent myocardial ischemia (Chun and Li, 1988; $\mathrm{Ai}$ and $\mathrm{Li}, 1992$ ), and also display antioxidant, antiatherosclerosis and antiphlegmonosis bioactivities (Renzulli et al., 2004; Kang et al., 2004; Liu et al., 2006). Thus, the differences in the content of active constituents between the two studied preparations were analyzed quantitatively by HPLC, in terms of four components: salvianolic acid $\mathrm{B}$, a hydrophilic compound; and, the lipophilics, cryptotanshinone, tanshinone I and tanshinone IIA. Of these analytes, however, marked differences were only demonstrated for salvianolic acid B, with no significant changes noted between preparation methods for the other three. The concentration of salvianolic acid B ranged up to $1.215 \mathrm{mg} / \mathrm{g}$ in the plant material samples prepared by nanotechnology after only 10 min of extraction; however, further increases were not noted with greater extraction time. By contrast, the concentration and extraction time of salvianolic acid B were associated in the traditionally powdered plant samples.

Based on the results described above, therefore, it appears reasonable to suggest that the release of the constituents will be more rapid and more complete in nanotechnology preparations of Chinese medicine plants compared to those prepared using the traditional methods. We also conclude that the improved antioxidant bioactivity demonstrated with the modern preparation resulting from higher salvianolic acid B content.

\section{Acknowledgment}

The authors are thankful to the National Science Council, Taiwan, ROC (NSC 95-2622-B-150-001-CC3) for financial support of the present research.

\section{References}

Ai, C.B., Li, L.N., 1992. Salvianolic acids D and E: two new depsides from Salvia miltiorrhiza. Planta Med. 58, 197-200.

Brucculeri, M., Kaplan, J., Lande, L., 2005. Reversal of citalopram-induced junctional bradycardia with intravenous sodium bicarbonate. Pharmacotherapy 25, 119-122.

Chun, B.A., Li, L.N., 1988. Stereostructure of salvianolic acid $\mathrm{B}$ and isolation of salvianolic acid C from Salvia miltiorrhiza. J. Nat. Prod. 51, 145-149.

Frenkel, K., 1992. Carcinogen-mediated oxidant formation and DNA damage. Pharmacol. Ther. 53, 127-166.

Gabel, A., Hinkelbein, J., 2004. Hypotensive cardio-circulatory failure and metabolic acidosis after suicidal intoxication with trimipramine and quetiapine. Der Anaesthesist $53,53-58$.

Halliwell, B., Gutteridge, J.M.C., 1984. Oxygen toxicity, oxygen radicals, transition metals, and disease. Biochem. J. 219, 1-4.

Imaida, K., Fukushima, T., Shivai, T., Ohtani, M., Nakanishi, K., Ito, N., 1983. Promoting activities of butylated hydroxyanisole and butylated hydroxytoluene on 2-stage urinary bladder carcinogenesis and inhibition of $\gamma$-glutamyl transpeptidase-positive foci development in the liver of rats. Carcinogenesis 4, 895-899.

Jovanovic, A.V., Flint, J.A., Varshney, M., Morey, T.E., Dennis, D.M., Duran, R.S., 2006. Surface modification of silica core-shell nanocapsules: biomedical implications. Biomacromolecules 7, 945-949.

Kagan, V.E., Bayir, H., Shvedova, A.A., 2005. Nanomedicine and nonatoxicology: two sides of the same coin. Nanomed.: Nanotechnol., Biol. Med. 1, 313-316.

Kang, D.G., Oh, H., Sohn, E.J., Hur, T.Y., Lee, K.C., Kim, K.J., Kim, T.Y., Lee, H.S., 2004. Lithospermic acid B isolated from Salvia miltiorrhiza ameliorates ischemia/ reperfusion-induced renal injury in rats. Life Sci. 75, 1801-1816.

Liu, G.T., Zhang, T.M., Wang, B.E., Wang, Y.W., 1992. Protective action of seven natural phenolic compounds against peroxidative damage to biomembranes. Biochem. Pharmacol. 43, 147-152.

Liu, J.R., Chen, M.J., Lin, C.W., 2005. Antimutagenic and antioxidant properties of milk-kefir and soymilk-kefir. J. Agric. Food Chem. 53, 2467-2474.

Liu, A.H., Li, L., Xu, M., Lin, Y.H., Guo, H.Z., Guo, D.A., 2006. Simultaneous quantification of six major phenolic acids in the roots of Salvia miltiorrhiza and four related traditional Chinese medicinal preparations by HPLC-DAD method. J. Pharm. Biomed. Anal. 41, 48-56.

Ma, Y.L., Henry, J.A., 2001. The antidotal effect of $a_{1}$-acid glycoprotein on amitriptyline toxicity in cardiac myocytes. Toxicology 169, 133-144.

Morey, T.E., Varshney, M., Flint, J.A., Rajasekaran, S., Shah, D.O., Dennis, D.M., 2004. Treatment of local anestheticinduced cardiotoxicity using drug scavenging nanoparticles. Nano Lett. 4, 757-759.

Pryor, W.A., 1991. The antioxidant nutrients and disease prevention - what do we know and what do we need to find out? Am. J. Clin. Nutr. 53, S391-S393. 
Renzulli, C., Galvano, F., Pierdomenico, L., Speroni, E., Guerra, M.C., 2004. Effects of rosmarinic acid against aflatoxin $\mathrm{B}_{1}$ and ochratoxin-A-induced cell damage in a human hepatoma cell line (Hep G2). J. Appl. Toxicol. 24, 289-296.

Shimada, K., Fujikawa, K., Yahara, K., Nakamura, T., 1992. Antioxidative properties of xanthan on the autoxidation of soybean oil in cyclodextrin emulsion. J. Agric. Food Chem. 40, 945-948.

Varshney, M., Morey, T.E., Shah, D.O., Flint, J.A., Moudgil, B.M., Seubert, C.N., Dennis, D.M., 2004. Pluronic micro- emulsions as nanoreservoirs for extraction of bupivacaine from normal saline. J. Am. Chem. Soc. 126, 5108-5112.

Wang, C.C., 2007. ROC patent pending (CP 22119).

Yen, G.C., Duh, P.D., Chuang, D.Y., 2000. Antioxidant activity of anthraquinones and anthrone. Food Chem. 70, 437-441.

Zajtchuk, R., 1999. New technologies in medicine: biotechnology and nanotechnology. Disease-a-Month 45, 453-495.

Zhou, L., Chow, M., Zuo, Z., 2006. Improved quality control method for Danshen products - consideration of both hydrophilic and lipophilic active components. J. Pharm. Biomed. Anal. 41, 744-750. 